\title{
A NOTE ON REDUCED JORDAN ALGEBRAS
}

\author{
KEVIN MCCRIMMON ${ }^{1}$
}

In this paper we give short proofs of two of the main theorems concerning reduced exceptional Jordan algebras $A=H\left(C_{3}, \gamma\right)$ : the AlbertJacobson Theorem that the Cayley coordinate algebra $C$ is determined by $A$ up to isomorphism, and the Springer Theorem that two such algebras $A, A^{\prime}$ are isomorphic if and only if they have isomorphic coordinate algebras and equivalent trace forms. We avoid using the generic norm by working directly with the reduced idempotents. Our proofs do not require that the algebras be exceptional, and are valid for arbitrary reduced simple algebras.

1. Reduced idempotents. Throughout this paper, $A$ will denote a Jordan algebra with identity element 1 over a field $\Phi$ of characteristic $\neq 2$. In this section we make no assumptions about the simplicity or finite-dimensionality of $A$. Recall that an idempotent $e$ is reduced if $A_{1}(e)=U_{e} A=\Phi e$. We assume the reader is familiar with the operators $U_{x}=2 L_{x}^{2}-L_{x^{2}}$ and the basic identities involving them (e.g. [3, pp. 1072-1075]).

Lemma 1 . Let $e$ be a reduced idempotent in $A, x=x_{1}+x_{1 / 2}+x_{0}\left(x_{i}\right.$ in the Peirce space $\left.A_{i}(e)\right)$ an element with $U_{e} x=x_{1}=\alpha e, U_{e} x^{2}=\beta e, U_{e} x_{1 / 2}^{2}$ $=\gamma e$ for $\beta, \gamma \neq 0$. Then $U_{x} e=\beta g, U_{x_{1 / 2}} e=\gamma f$ where $g, f$ are reduced idempotents with $f$ orthogonal to $e$ and $g$ of the form $g=\rho e+y+(1-\rho) f$ with $\rho=\alpha^{2} \beta^{-1}, y=\alpha \beta^{-1} x_{1 / 2}$ an element in $A_{1 / 2}(e, f)$ satisfying $y^{2}$ $=\rho(1-\rho)(e+f)$ since $x_{1 / 2} \in A_{1 / 2}(e, f)$ has $x_{1 / 2}^{2}=\gamma(e+f)$.

Proof. Since $U_{e} U_{x}(\beta g)=U_{e} U_{x^{2} e}=\left(U_{e} x^{2}\right)^{2}=\beta^{2} e^{2} \neq 0$, we see $g \neq 0$, and $\beta^{2} g^{2}=\left(U_{x} e\right)^{2}=U_{x} U_{e} x^{2}=\beta U_{x} e=\beta^{2} g$ shows $g$ is an idempotent. It is reduced since $\beta^{2} U_{g} A=U_{U(x) e} A=U_{x} U_{e} U_{x} A \subset U_{x}(\Phi e)=\Phi g$. Repeating this argument for $x_{1 / 2}$, we get $U_{x_{1 / 2}} e=\gamma f$ for $f$ a reduced idempotent, which is orthogonal to $e$ since $U_{x_{1 / 2}} A_{1}(e) \subset A_{0}(e)$. Then $U_{x} e=\alpha^{2} U_{e} e+2 \alpha\left\{e e x_{1 / 2}\right\}+U_{x_{1 / 2}} e=\alpha^{2} e+\alpha x_{1 / 2}+\gamma f=\beta\{\rho e+y+(1-\rho) f\}$ since $\gamma=\beta-\alpha^{2}$ follows from $\beta e=U_{e} x^{2}=\alpha^{2} e+U_{e} x_{1 / 2}^{2}=\left(\alpha^{2}+\gamma\right) e$. Since $x_{1 / 2}^{2}=U_{e} x_{1 / 2}^{2}+U_{1-e} x_{1 / 2}^{2}=\gamma e+U_{x_{1 / 2}} e=\gamma(e+f)$ and $\rho(1-\rho)=\alpha^{2} \gamma \beta^{-2}$, we have $y^{2}=\rho(1-\rho)(e+f)$. Since $2 \gamma f \cdot x_{1 / 2}=2 x_{1 / 2} \cdot U_{x_{1 / 2}} e=2\left\{x_{1 / 2} e x_{1 / 2}^{2}\right\}$ $=2\left\{x_{1 / 2} e \gamma e\right\}=\gamma x_{1 / 2}$, we have $x_{1 / 2} \in A_{1 / 2}(e) \cap A_{1 / 2}(f)=A_{1 / 2}(e, f)$.

The next lemma is a variant of a result of N. Jacobson $[2$, p. 82].

Received by the editors April 7, 1967.

${ }^{1}$ This research was partially supported by AFOSR Grant No. 335-63. 
If $e$ is an idempotent, then $c=2 e-1$ satisfies $c^{2}=1$, so $U_{c}$ is an automorphism of $A$ of period two. This automorphism will be called the Peirce reflection $S_{e}$ determined by $e ; S_{e}$ acts as the identity $I$ on the Peirce spaces $A_{1}(e)$ and $A_{0}(e)$, and as $-I$ on $A_{1 / 2}(e)$.

Lemma 2. Let $e_{1}, e_{2}$ be reduced idempotents in $A$. Then

(a) There is a $\rho \in \Phi$, called the projection coefficient of $e_{1}$ and $e_{2}$, such that $U_{e_{1}} e_{2}=\rho e_{1}$ and $U_{e_{2}} e_{1}=\rho e_{2}$.

(b) If $\rho=1$, then $e=\frac{1}{4}\left(e_{1}+e_{2}\right)^{2}$ is idempotent and the Peirce reflection $S_{e}$ maps $e_{1}$ onto $e_{2}$.

(c) If $\rho=0$, then $g=e_{1} \cdot\left(e_{1}-e_{2}\right)$ is idempotent and $S_{g}$ maps $e_{1}$ onto the reduced idempotent $f_{1}=e_{1} \cdot\left(e_{1}-2 e_{2}\right)$, which is orthogonal to $e_{2}$.

(d) If $\rho \neq 0,1$, there are reduced idempotents $f_{i}$ orthogonal to $e_{i}$ $(i=1,2)$ with $h=e_{1}+f_{1}=e_{2}+f_{2}$ an idempotent such that for some $y \in A_{1}(h)$ the element $s=(1-h)+y$ is invertible and $U_{s} e_{1}=\rho e_{2}, U_{s} f_{1}=\rho f_{2}$.

Proof. We have $U_{e_{i}} e_{j}=\rho_{i} e_{i}$ for some $\rho_{i}(i \neq j)$. Since $\rho_{1}^{3} e_{1}=U_{\rho_{1} e_{1}} e_{2}$ $=U_{U\left(e_{1}\right) e_{2}} e_{2}=U_{e_{1}} U_{e_{2}} U_{e_{1}} e_{2}=\rho_{1} \rho_{2} \rho_{1} e_{1}$ we have $\rho_{1}^{2}\left(\rho_{1}-\rho_{2}\right)=0$; similarly $\rho_{2}^{2}\left(\rho_{2}-\rho_{1}\right)=0$, so either $\rho_{1}-\rho_{2}=0$ or $\rho_{1}^{2}=\rho_{2}^{2}=0$, and in either case $\rho_{1}=\rho_{2}=\rho$. We claim the subalgebra generated by $e_{1}, e_{2}$ is $\Phi\left[e_{1}, e_{2}\right]$ $=\Phi e_{1}+\Phi x+\Phi e_{2}$, where

$$
x=2 e_{1} \cdot e_{2} \quad e_{i} \cdot x=\rho e_{i}+\frac{1}{2} x \quad x^{2}=\rho\left(e_{1}+x+e_{2}\right) .
$$

Indeed, such an $x$ has $e_{i} \cdot x=2 e_{i} \cdot\left(e_{i} \cdot e_{j}\right)=U_{e_{i}} e_{j}+e_{i}^{2} \cdot e_{j}=\rho e_{i}+e_{i} \cdot e_{j}$, by $\left[3\right.$, p. 1073, (10)] and $x^{2}=U_{e_{1}} e_{2}^{2}+U_{e_{2}} e_{1}^{2}+2 e_{1} \cdot U_{e_{2}} e_{1}=\rho e_{1}+\rho e_{2}$ $+2 \rho e_{1} \cdot e_{2}$.

If $\rho=1$, set $e=\frac{1}{4}\left(e_{1}+e_{2}+x\right), z=\frac{1}{2}\left(e_{1}-e_{2}\right), z^{2}=\frac{1}{4}\left(e_{1}+e_{2}-x\right)$. Then we see $e^{2}=e, e \cdot z=\frac{1}{2} z, e \cdot z^{2}=0$. Thus $e$ is an idempotent with $z \in A_{1 / 2}(e)$, $z^{2} \in A_{0}(e)$. Since $e_{1}=e+z+z^{2}, e_{2}=e-z+z^{2}$, this shows $S_{e} e_{1}=e_{2}$.

If $\rho=0$, set $g=e_{1} \cdot\left(e_{1}-e_{2}\right)=e_{1}-\frac{1}{2} x, f_{1}=e_{1} \cdot\left(e_{1}-2 e_{2}\right)=e_{1}-x$. Then $x^{2}=0, g^{2}=g, g \cdot x=\frac{1}{2} x$ imply $g$ is an idempotent with $x \in A_{1 / 2}(g)$. Since $e_{1}=g+\frac{1}{2} x, f_{1}=g-\frac{1}{2} x$, this shows $S_{g} e_{1}=f_{1}$. Because $S_{g}$ is an automorphism, we know $f_{1}$ is a reduced idempotent, and $e_{2} \cdot f_{1}=e_{2} \cdot e_{1}-e_{2} \cdot x=0$.

If $\rho \neq 0,1$, set $x_{i}=x-2 \rho e_{i}(i=1,2)$. Then $e_{i} \cdot x_{i}=\frac{1}{2} x_{i}$, so $x_{i} \in A_{1 / 2}\left(e_{i}\right)$, and $x_{i}^{2}=\rho\left(e_{i}+e_{j}-x\right)$, so $U_{e_{i}} x_{i}^{2}=\rho U_{e_{i}}\left(e_{i}+e_{j}-x\right)=\rho(1-\rho) e_{i} \neq 0$. Applying Lemma 1, $U_{x_{i}} e_{i}=\rho(1-\rho) f_{i}$ where $f_{i}$ is a reduced idempotent orthogonal to $e_{i}$ such that $x_{i} \in A_{1 / 2}\left(e_{i}, f_{i}\right)$ has $x_{i}^{2}=\rho(1-\rho)\left(e_{i}+f_{i}\right)$. But $x_{1}^{2}=\rho\left(e_{1}+e_{2}-x\right)=x_{2}^{2}$, so $e_{1}+f_{1}=e_{2}+f_{2}=h$. Also, $(1-\rho)\left(e_{i}+f_{i}\right)=\rho^{-1} x_{i}^{2}$ $=e_{i}+e_{j}-x=e_{i}-x_{i}-2 \rho e_{i}+e_{j}$, so $e_{j}=\rho e_{i}+x_{i}+(1-\rho) f_{i}$. Set $y=\rho\left(e_{1}-f_{1}\right)+x_{1}$; then $y^{2}=\rho^{2}\left(e_{1}+f_{1}\right)+\rho(1-\rho)\left(e_{1}+f_{1}\right)=\rho h$, so $y$ is invertible in $A_{1}(h), \quad$ and $U_{y} e_{1}=\rho^{2} e_{1}+\rho(1-\rho) f_{1}+\rho x_{1}=\rho e_{2}, \quad U_{y} f_{1}$ $=U_{y}\left(h-e_{1}\right)=y^{2}-U_{y} e_{1}=\rho\left(h-e_{2}\right)=\rho f_{2}$. If $s=(1-h)+y$ then $s$ is invertible in $A$ and $U_{s}=U_{y}$ on $A_{1}(h)$, so $U_{s} e_{1}=\rho e_{2}, U_{s} f_{1}=\rho f_{2}$. 
2. Peirce quadratic forms. If $e_{1}, e_{2}$ are reduced orthogonal idempotents then the Peirce quadratic form $Q_{e_{1}, e_{2}}$ determined by $e_{1}$ and $e_{2}$ is the generic norm of the Peirce subalgebra $A_{1}\left(e_{1}+e_{2}\right)=\Phi e_{1}+A_{12}$ $+\Phi e_{2}$. Thus $Q\left(\alpha_{1} e_{1}+a_{12}+\alpha_{2} e_{2}\right)=\alpha_{1} \alpha_{2}-Q_{0}\left(a_{12}\right)$ where $a_{12}^{2}=Q_{0}\left(a_{12}\right)\left(e_{1}+e_{2}\right)$. A similarity of Peirce quadratic forms $Q=Q_{e_{1}, e_{2}}$ in $A$ and $Q^{\prime}=Q e_{1}^{\prime} e_{2}^{\prime}$ in $A^{\prime}$ is a bijection $S$ of $A_{1}\left(e_{1}+e_{2}\right)$ onto $A_{1}^{\prime}\left(e_{1}+e_{2}\right)$ satisfying $Q^{\prime}(S x)$ $=\sigma Q(x)$ for all $x \in A_{1}\left(e_{1}+e_{2}\right)$ and such that $S e_{i}=\sigma_{i} e_{i}^{\prime}$ for some nonzero scalars $\sigma_{1}, \sigma_{2}\left(\sigma=\sigma_{1} \sigma_{2}\right)$. The main source of similarities is the following

Lemma 3. Suppose $e_{1}, e_{2}$ are reduced orthogonal idempotents in $A$, and similarly for $f_{1}, f_{2}$. If $s$ is invertible and $U_{s} e_{i}=\sigma_{i} f_{i}(i=1,2)$ then $S=U_{s}$ is a similarity of $Q_{e_{1}, e_{2}}$ with $Q_{f_{1}, f_{2}}$ with ratio $\sigma=\sigma_{1} \sigma_{2}$.

Proof. Clearly $U_{s}$ is a similarity of $\Phi e_{1}+\Phi e_{2}$ onto $\Phi f_{1}+\Phi f_{2}$, with ratio $\sigma$, so it suffices to check it from $A_{1 / 2}\left(e_{1}, e_{2}\right)$ to $A_{1 / 2}\left(f_{1}, f_{2}\right)$. The formula $U_{s} U_{e_{1}, e_{2}} A=U_{s} U_{U\left(s^{-1}\right) f_{1}, U\left(s^{-1}\right) f_{2}} A=U_{s} U_{s}^{-1} U_{f_{1}, f_{2}} U_{s}^{-1} A=U_{f_{1}, f_{2}} A$ show that $U_{s}$ is a bijection of $A_{1 / 2}\left(e_{1}, e_{2}\right)$ on to $A_{1 / 2}\left(f_{1}, f_{2}\right)$. The component of $s^{2}$ in $A_{1}\left(e_{1}+e_{2}\right)$ is $U_{e_{1}+e_{2}} s^{2}=U_{U\left(s^{-1}\right)\left(\sigma_{1} f_{1}+\sigma_{2} f_{2}\right)} U_{s} 1$ $=U_{s}^{-1} U_{\sigma_{1} f_{1}+\sigma_{2} f_{2}} U_{s}^{-1} U_{s} 1=U_{s}^{-1}\left(\sigma_{1}^{2} f_{1}+\sigma_{2}^{2} f_{2}\right)=\sigma_{1} e_{1}+\sigma_{2} e_{2}$, and hence for $a \in A_{1 / 2}\left(e_{1}, e_{2}\right) \subset A_{1}\left(e_{1}+e_{2}\right)$ we have $\left(U_{s} a\right)^{2}=U_{s} U_{a} s^{2}=U_{s} U_{a} U_{e_{1}+e_{2}} s^{2}$ $=U_{s} U_{a}\left(\sigma_{1} e_{1}+\sigma_{2} e_{2}\right)=U_{s}\left\{\sigma_{1} U_{e_{2}} a^{2}+\sigma_{2} U_{e_{1}} a^{2}\right\}=Q_{0}(a) U_{s}\left\{\sigma_{1} e_{2}+\sigma_{2} e_{1}\right\}$ $=Q_{0}(a)\left\{\sigma_{1} \sigma_{2} f_{2}+\sigma_{2} \sigma_{1} f_{1}\right\}=\sigma Q_{0}(a)\left(f_{1}+f_{2}\right)$. Thus $Q_{0}\left(U_{s} a\right)=\sigma Q_{0}(a)$, and $U_{s}$ is a similarity with ratio $\sigma$.

Recall that two orthogonal idempotents $e_{1}, e_{2}$ are connected if there is an element $y \in A_{1 / 2}\left(e_{1}, e_{2}\right)$ which is invertible in $A_{1}\left(e_{1}+e_{2}\right)$.

Theorem 1. If $A$ is a Jordan algebra in which any two reduced orthogonal idempotents are connected, then any two Peirce quadratic forms in $A$ are similar, and the similarity may be taken in the group $\mathcal{u}(A)$ generated by the $U_{s}$ for s invertible.

Proof. We write $Q \sim Q^{\prime}$ if such a similarity exists; this defines an equivalence relation. The connectivity assumption guarantees

$$
Q_{e_{1}, e_{2}} \sim Q_{e_{2}, e_{1}} \text { if } e_{1} \perp e_{2} \text { are orthogonal, }
$$

since there is an element $y \in A_{1 / 2}\left(e_{1}, e_{2}\right)$ with $y^{2}=\sigma\left(e_{1}+e_{2}\right)$ for $\sigma \neq 0$, and $s=\left(1-e_{1}-e_{2}\right)+y$ is invertible in $A$ with $U_{s} e_{i}=U_{y} e_{i}=U_{e_{j}} y^{2}=\sigma e_{j}$ $(i \neq j)$, so we can apply the lemma. Using this same $U_{s}$ we see

$$
Q_{e_{0}, e_{1}} \sim Q_{e_{0}, e_{2}} \text { if } e_{0} \perp e_{1} \perp e_{2}
$$

since $U_{s} e_{0}=e_{0}, U_{s} e_{1}=\sigma e_{2}$.

We use this to establish the more general case

$$
Q_{e_{0}, e .} \sim Q_{e_{0}, e_{2}} \text { if } e_{0} \perp e_{1}, e_{0} \perp e_{2} .
$$


Let $\rho$ be the projection coefficient of $e_{1}$ and $e_{2}$. If $\rho=1$, there is a Peirce reflection, viz. the $S_{e}$ of Lemma $2\left(e \in \Phi\left[e_{1}, e_{2}\right] \subset A_{0}\left(e_{0}\right)\right)$, with $S_{e} e_{1}=e_{2}$, $S_{e} e_{0}=e_{0}$, so $Q_{e_{0}, e_{1}} \sim Q_{e_{0}, e_{2}}$ by Lemma 3 (or directly, since $S_{e}$ is an automorphism). If $\rho=0$ then $S_{g} e_{1}=f_{1} \perp e_{2}, S_{g} e_{0}=e_{0}$ so $Q_{e_{0}, e_{1}} \sim Q_{e_{0}, f_{1}} \sim Q_{e_{0}, e_{2}}$ (where the last step follows from (2) since $e_{0} \perp f_{1} \perp e_{2}$ ). If $\rho \neq 0,1$, there is $U_{s} \in \mathcal{U}(A)$ with $U_{s} e_{0}=e_{0}, U_{s} e_{1}=\rho e_{2}$ and again $Q_{e_{0}, e_{1}} \sim Q_{e_{0}, e_{2}}$ by Lemma 3.

Finally, we turn to the general case:

$$
Q_{e_{1}, e_{2}} \sim Q_{f_{1}, f_{2}} \text { if } e_{1} \perp e_{2}, f_{1} \perp f_{2} .
$$

Let $\rho$ be the projection coefficient of $e_{1}$ and $f_{1}$. If $\rho=1$, then $S_{e} e_{1}=f_{1}$, so $Q_{e_{1}, e_{2}} \sim Q_{S e_{1}, S e_{2}}=Q_{f_{1}, S e_{2}} \sim Q_{f_{1}, f_{2}}$ (using (3) for the last step). If $\rho=0$ we have $S_{g} e_{1}=g_{1} \perp f_{1}$ so $Q_{e_{1}, e_{2}} \sim Q_{S e_{1}, S e_{2}}=Q_{\theta_{1}, S e_{2}} \sim Q_{g_{1}, f_{1}} \sim Q_{f_{1}, g_{1}} \sim Q_{f_{1}, f_{2}}$ (using (3), (1), (3) for the last steps). If $\rho \neq 0,1$ we have $e_{1} \perp g_{1}, f_{1} \perp h_{1}$ and $U_{s} \in \mathcal{u}(A)$ with $U_{s} e_{1}=\rho f_{1}, U_{s} g_{1}=\rho h_{1}$, and hence $Q_{e_{1}, e_{2}} \sim Q_{e_{1}, \theta_{1}}$ $\sim Q_{f_{1}, h_{1}} \sim Q_{f_{1}, f_{2}}$ by (3), Lemma 3, and (3) again.

As an immediate corollary we have

Theorem 2 (Albert-Jacobson [1]). If two reduced simple Jordan matrix algebras $A=H\left(D_{n}, \gamma\right)$ and $A^{\prime}=H\left(D_{n}^{\prime}, \gamma^{\prime}\right)$ are isomorphic ( $n \geqq 2, D$ and $D^{\prime}$ composition algebras) then $D$ and $D^{\prime}$ are isomorphic.

Proof. By simplicity, any two reduced orthogonal idempotents of $A$ (or $A^{\prime}$ ) are connected. Hence, by Theorem 1, any two Peirce quadratic forms of $A$ (or $A^{\prime}$ ) are similar. The isomorphism of $A$ and $A^{\prime}$ implies that for any Peirce quadratic form of $A$ there is a similar one of $A^{\prime}$. Consequently, the Peirce quadratic forms of $A$ are similar to those of $A^{\prime}$. Taking in $A$ the diagonal idempotents $e_{11}, e_{22}$, the corresponding Peirce quadratic form is the sum of a hyperbolic 2-dimensional quadratic form and of a multiple of the norm form $N$ of $D$. Similarly in $A^{\prime}$. Consequently, Witt's theorem implies that the norm forms $N$ and $N^{\prime}$ of $D$ and $D^{\prime}$ are similar. Then $D$ and $D^{\prime}$ are known to be isomorphic (see [1]).

3. Reduced algebras of degree three. Throughout this section, we will be concerned with reduced simple algebras $H\left(D_{3}, \gamma\right)$ of degree three, $D$ a composition algebra. Given a reduced idempotent $e_{1}$, we can write $1-e_{1}=e_{2}+e_{3}$, where $e_{2}, e_{3}$ are reduced orthogonal idempotents. The set of values $Q_{0}\left(A_{23}\right)=-Q_{e_{2}, e_{3}}\left(A_{23}\right)$ of $Q_{0}$ on $A_{23}$ is called the norm class of $e_{1}$, and is denoted by $K\left(e_{1}\right)$. By Witt's Theorem, it is independent of the decomposition of $1-e_{1}$ into reduced idempotents, since $Q$ is nondegenerate on $A_{1}\left(1-e_{1}\right)$ by simplicity. The nomenclature is justified by 


$$
K\left(e_{i}\right)=\gamma_{k}^{-1} \gamma_{j} N(D) \text { if } e_{l}=e_{l l} \text { in } H\left(D_{3}, \gamma\right),
$$

since $A_{j k}=D[j k], x[j k]^{2}=\gamma_{\mathbf{k}}^{-1} \gamma_{j} N(x)$. From this we immediately see

$$
K\left(e_{i}\right)=K\left(e_{j}\right) K\left(e_{k}\right) \quad \text { if } 1=e_{i}+e_{j}+e_{k} .
$$

Theorem 3. If $A, A^{\prime}$ are reduced simple Jordan algebras of degree three with equivalent trace forms and isomorphic coordinate algebras, then any two reduced idempotents $e, e^{\prime}$ with the same norm class $K(e)$ $=K\left(e^{\prime}\right)$ may be mapped into each other under an isomorphism of $A$ onto $A^{\prime}$.

Proof. Let $1=e_{1}+e_{2}+e_{3}, 1^{\prime}=e_{1}^{\prime}+e_{2}^{\prime}+e_{3}^{\prime}$ for $e_{i}, e_{i}^{\prime}$ reduced and $e=e_{1}, e^{\prime}=e_{1}^{\prime}$. By the Coordinatization Theorem [3, p. 1077] there are isomorphisms $A \cong H\left(D_{3}, \gamma\right), A^{\prime} \cong H\left(D_{3}^{\prime}, \gamma^{\prime}\right)$ mapping $e_{i}, e_{i}^{\prime}$ in to the diagonal idempotents $e_{i i}, e_{i i}^{\prime}$; we may assume $D=D^{\prime}$. Here $\gamma=\left(1, \gamma_{2}, \gamma_{3}\right)$, $\gamma^{\prime}=\left(1^{\prime}, \gamma_{2}^{\prime}, \gamma_{3}^{\prime}\right)$, where $K(e)=\gamma_{3}^{-1} \gamma_{2} N(D)=\gamma_{3}^{\prime-1} \gamma_{2}^{\prime} N(D)=K\left(e^{\prime}\right)$. Since $\tau(x[23], \quad x[23])=2 \gamma_{3}^{-1} \gamma_{2} N(x)$ and $\tau^{\prime}\left(x[23]^{\prime}, \quad x[23]^{\prime}\right)$ $=2 \gamma_{3}^{\prime-1} \gamma_{2}^{\prime} N(x)$, the generic trace forms on $D[23]$ and $D[23]^{\prime}$ are equivalent, and this extends to an equivalence of $V^{\prime}=\Phi e_{1}^{\prime}+\Phi e_{2}^{\prime}$ $+\Phi e_{3}^{\prime}+D[23]^{\prime}$ on to $V=\Phi e_{1}+\Phi e_{2}+\Phi e_{3}+D[23]$. Since $\tau$ and $\tau^{\prime}$ are equivalent by hypothesis, Witt's Theorem furnishes us with an equivalence of $V^{\perp \perp}=D[12]^{\prime}+D[13]^{\prime}$ on to $V^{\perp}=D[12]+D[13]$. Since $\tau^{\prime}\left(1[31]^{\prime}, 1[31]^{\prime}\right)=2 \gamma_{3}^{\prime}$, there must be an element $u=x[21]+y[31]$ $(x, y \in D)$ with $\tau(u, u)=2\left\{\gamma_{2} N(x)+\gamma_{3} N(y)\right\}=2 \gamma_{3}^{\prime}$. Then $U_{e_{1}} u^{2}$ $=\gamma_{3}^{\prime} e_{1} \neq 0$, and by Lemma $1, U_{u} e_{1}=\gamma_{3}^{\prime} f_{1}$ where $f_{1}$ is a reduced idempotent orthogonal to $e_{1}$ (because $\left.u \in A_{1 / 2}\left(e_{1}\right)\right)$ with $u \in A_{1 / 2}\left(e_{1}, f_{1}\right)$ and $u^{2}=\gamma_{3}^{\prime}\left(e_{1}+f_{1}\right)$. Then $1=e_{1}+f_{1}+g_{1}$ for $g_{1}$ reduced and $K\left(g_{1}\right)=\gamma_{3}^{\prime} N(D)$. We already had $K\left(e_{1}\right)=K\left(e_{1}^{\prime}\right)=\gamma_{3}^{\prime-1} \gamma_{2}^{\prime} N(D)$, hence $K\left(f_{1}\right)=K\left(e_{1}\right) K\left(g_{1}\right)$ $=\gamma_{2}^{\prime} N(D)$ by (6). But this means that we can choose $u_{12} \in A_{1 / 2}\left(e_{1}, g_{1}\right)$, $u_{13} \in A_{1 / 2}\left(e_{1}, f_{1}\right)$ with $u_{12}^{2}=\gamma_{2}^{\prime-1}\left(e_{1}+g_{1}\right), u_{13}^{2}=\gamma_{3}^{\prime-1}\left(e_{1}+f_{1}\right)$, and by the Coordinatization Theorem there is an isomorphism $A \cong H\left(D_{3}, \gamma^{\prime}\right)$ for $\gamma^{\prime}=\left(1, \gamma_{2}^{\prime}, \gamma_{3}^{\prime}\right)$ sending $e_{1} \rightarrow e_{11}^{\prime}, g_{1} \rightarrow e_{22}^{\prime}, f_{1} \rightarrow e_{33}^{\prime}$. Thus $A \cong H\left(D_{3}, \gamma^{\prime}\right)$ $\cong A^{\prime}$ under isomorphisms mapping $e=e_{1} \leftrightarrow e_{11}^{\prime} \leftrightarrow e_{1}^{\prime}=e^{\prime}$.

COROLlary. Two reduced idempotents $e, e^{\prime}$ in a reduced simple Jordan algebra of degree three are conjugate under the group of automorphisms if and only if $K(e)=K\left(e^{\prime}\right)$.

TheOREM 4 (SPRINGER [4]). Two reduced simple Jordan algebras of degree three are isomorphic if and only if they have isomorphic coordinate algebras and equivalent trace forms.

Proof. The conditions are necessary by the Albert-Jacobson Theorem. The previous theorem shows they will be sufficient if they allow us to find reduced idempotents in $A$ and $A^{\prime}$ with the same norm class. 
Let $1^{\prime}=e_{1}^{\prime}+e_{2}^{\prime}+e_{3}^{\prime}$ where $e^{\prime}=e_{1}^{\prime}$ has $K\left(e^{\prime}\right)=\gamma_{3}^{\prime-1} \gamma_{2}^{\prime} N\left(D^{\prime}\right)$ and $A^{\prime}=H\left(D_{3}^{\prime}, \gamma^{\prime}\right)$ for $\gamma^{\prime}=\left(1, \gamma_{2}^{\prime}, \gamma_{3}^{\prime}\right)$. We must find $e \in A$ with $K(e)$ $=K\left(e^{\prime}\right)$.

Choose a (temporary) coordinatization $A=H\left(D_{3}, \gamma\right)$ with $1=e_{1}$ $+e_{2}+e_{3}, \gamma=\left(\gamma_{1}, \gamma_{2}, \gamma_{3}\right)$ (to have complete symmetry among the indices we do not assume $\gamma_{1}=1$ ). Trivially the trace forms $\tau$ on $\Phi e_{1}+\Phi e_{2}+\Phi e_{3}$ and $\tau^{\prime}$ on $\Phi e_{1}^{\prime}+\Phi e_{2}^{\prime}+\Phi e_{3}^{\prime}$ are equivalent, so if $\tau$ and $\tau^{\prime}$ are equivalent we can use Witt's Theorem again to obtain an equivalence of $D[12]+D[13]+D[23]$ with $D^{\prime}[12]^{\prime}+D^{\prime}[13]^{\prime}+D^{\prime}[23]^{\prime}$. Since $\tau^{\prime}\left(1[23]^{\prime}, 1[23]^{\prime}\right)=2 \gamma_{3}^{\prime-1} \gamma_{2}^{\prime}$, there is an element $u=x[12]$ $+y[23]+z[31]$ with $\tau(u, u)=2\left\{\gamma_{2}^{-1} \gamma_{1} N(x)+\gamma_{3}^{-1} \gamma_{2} N(y)+\gamma_{1}^{-1} \gamma_{3} N(z)\right\}$ $=2 \gamma_{3}^{\prime-1} \gamma_{2}^{\prime}$.

We cannot have $N(x)=N(y)=N(z)=0$. First suppose that two of the norms are zero; by symmetry we may assume $N(x)=N(z)=0$. Then $\gamma_{3}^{-1} \gamma_{2} N(y)=\gamma_{3}^{\prime-1} \gamma_{2}^{\prime}$ implies $K\left(e_{1}\right)=K\left(e^{\prime}\right)$, and we may take $e=e_{1}$.

Suppose only one of the norms is zero, say $N(y)=0$. Then $v=x[12]$ $+z[31]$ has $\tau(v, v)=\tau(u, u)=2 \gamma_{3}^{\prime-1} \gamma_{2}^{\prime}$. Since $N(x), N(z) \neq 0$ we can re-coordinatize $A$ (keeping the same idempotents $e_{1}, e_{2}, e_{3}$ ) in such a way that $v$ has the form $v=1[21]+1[31]$ in $H\left(D_{3}, \gamma\right)$ for some new $\gamma=\left(1, \gamma_{2}, \gamma_{3}\right)$. Then $U_{e_{1}} v^{2}=\frac{1}{2} \tau(v, v) e_{1}=\gamma_{3}^{\prime-1} \gamma_{2}^{\prime} e_{1} \neq 0$. By Lemma 1 we have $U_{v} e_{1}=\gamma_{3}^{\prime-1} \gamma_{2}^{\prime} f_{1}$ where $f_{1}$ is a reduced idempotent orthogonal to $e_{1}$ with $v \in A_{1 / 2}\left(e_{1}, f_{1}\right)$ and $v^{2}=\gamma_{3}^{\prime-1} \gamma_{2}^{\prime}\left(e_{1}+f_{1}\right)$. This implies $g_{1}=1-e_{1}$ $-f_{1}$ has $K\left(g_{1}\right)=\gamma_{3}^{\prime-1} \gamma_{2}^{\prime} N(D)=\gamma_{3}^{\prime-1} \gamma_{2}^{\prime} N\left(D^{\prime}\right)=K\left(e^{\prime}\right)$, and we may take $e=g_{1}$.

Finally, suppose none of the norms is zero. Then again we can re-coordinatize $A$ in such a way that $u$ has the form $u=1[21]+1[31]$ $+x[23]$ in $H\left(D_{3}, \gamma\right)$ for some new $\gamma=\left(1, \gamma_{2}, \gamma_{3}\right)$ and some new $x \in D$. Here $\tau(u, u)=2\left\{\gamma_{2}+\gamma_{3}+\gamma_{2} \gamma_{3} N(y)\right\}=2 \gamma_{3}^{\prime-1} \gamma_{2}^{\prime}$ for $y=\gamma_{3}^{-1} x$ (and $\gamma_{2}+\gamma_{3} \neq 0$ ). Since $y$ is invertible in $D$, set $v=e_{1}+\gamma_{2}^{-1} y^{-1}[21]$ $+\gamma_{3}^{-1} y^{-1}[31]=e_{1}+v_{1 / 2}$. Then $U_{e_{1}} v^{2}=\beta e_{1}, U_{e_{1}} v_{1 / 2}^{2}=\delta e_{1}$ for $\beta=1$ $+\gamma_{2}^{-2} \gamma_{2} N\left(y^{-1}\right)+\gamma_{3}^{-2} \gamma_{3} N\left(y^{-1}\right)=\gamma_{3}^{\prime-1} \gamma_{2}^{\prime}\left\{\gamma_{2} \gamma_{3} N(y)\right\}^{-1} \neq 0$ and $\delta$ $=\left(\gamma_{2}^{-1}+\gamma_{3}^{-1}\right) N\left(y^{-1}\right)=\left(\gamma_{2}+\gamma_{3}\right)\left\{\gamma_{2} \gamma_{3} N(y)\right\}^{-1} \neq 0$. Applying Lemma 1, $U_{v} e_{1}=\beta g_{1}$ and $U_{v_{1 / 2}} e_{1}=\delta f_{1}$ for $e_{1}, f_{1}, g_{1}$ reduced, $e_{1}$ and $f_{1}$ orthogonal, and $g_{1} \in A_{1}\left(e_{1}+f_{1}\right)$. Thus there is another reduced idempotent $h_{1}$ orthogonal to $g_{1}$ with $g_{1}+h_{1}=e_{1}+f_{1}$. If $1=e_{1}+f_{1}+k_{1}=g_{1}+h_{1}+k_{1}$ then $k_{1}$ is reduced. Now $v$ is invertible in $A_{1}\left(e_{1}+f_{1}\right)$ because $v^{2}-v=\delta\left(e_{1}+f_{1}\right)$, so $s=k_{1}+v$ is invertible in $A$, and $U_{s} k_{1}=k_{1}, U_{8} e_{1}=U_{v} e_{1}=\beta g_{1}$. From Lemma 3 we see $U_{s}$ is an equivalence of $Q_{k_{1}, g_{1}}$ with $\beta Q_{k_{1}, e_{1}}$. Thus $K\left(h_{1}\right)=\beta K\left(f_{1}\right)$. Applying (6) twice, $K\left(g_{1}\right)=K\left(h_{1}\right) K\left(k_{1}\right)=\beta K\left(f_{1}\right) K\left(k_{1}\right)$ $=\beta K\left(e_{1}\right)$. But $\beta K\left(e_{1}\right)=\gamma_{3}^{\prime-1} \gamma_{2}^{\prime}\left\{\gamma_{2} \gamma_{3} N(y)\right\}^{-1} \gamma_{3}^{-1} \gamma_{2} N(D)=\gamma_{3}^{\prime-1} \gamma_{2}^{\prime} N(D)$ $=K\left(e^{\prime}\right)$, and again we can take $e=g_{1}$.

REMARK. As the referee has kindly pointed out, most of the above 
results can be extended to the case of algebras $A, A^{\prime}$ over different ground fields $\Phi, \Phi^{\prime}$. In Theorem 2, an isomorphism of $A$ and $A^{\prime}$ as simple rings induces an isomorphism of their centers $\Phi$ and $\Phi^{\prime}$, and the argument shows that the coordinate algebras $D$ and $D^{\prime}$ are semilinearly isomorphic. In Theorem 3 , if $A$ and $A^{\prime}$ have trace forms which are semilinearly equivalent and coordinate algebras which are semilinearly isomorphic relative to some given isomorphism of the ground fields $\Phi$ and $\Phi^{\prime}$, and $e, e^{\prime}$ are reduced idempotents whose norm classes correspond under the field isomorphism, then $e$ and $e^{\prime}$ may be mapped in to each other under a semilinear isomorphism of $A$ on to $A^{\prime}$. Then Theorem 4 says that two reduced simple Jordan algebras of degree 3 are (ring) isomorphic if and only if they have trace forms which are semilinearly equivalent and coordinate algebras which are semilinearly isomorphic relative to a fixed isomorphism of the ground fields.

\section{REFERENCES}

1. A. A. Albert and N. Jacobson, On reduced exceptional simple Jordan algebras, Ann. of Math. (2) 66 (1957), 400-417.

2. N. Jacobson, Some groups of transformations defined by Jordan algebras. II, J. Reine Angew. Math. 204 (1960), 74-98.

3. K. McCrimmon, A general theory of Jordan rings, Proc. Nat. Acad. Sci. U.S.A. 56 (1966), 1072-1079.

4. T. A. Springer, The classification of reduced exceptional simple Jordan algebras, Nederl. Akad. Wetensch. Proc. Ser. A 63 (1960), 414-422.

Massachusetts Institute of Technology and UNIVERSITY OF VIRGINIA 